\title{
Global myocardial perfusion and diastolic function are impaired to a similar extent in patients with type 2 diabetes mellitus and in patients with coronary artery disease - evaluation by contrast echocardiography and pulsed tissue Doppler
}

\author{
V. Dounis • T. Siegmund $\cdot$ A. Hansen - J. Jensen • \\ P.-M. Schumm-Draeger $\cdot$ H. von Bibra \\ Received: 25 April 2006 / Accepted: 19 June 2006 / Published online: 21 September 2006 \\ (C) Springer-Verlag 2006
}

\begin{abstract}
Aims/hypothesis Using modern echocardiography, we quantified the extent of global myocardial function and perfusion abnormalities in patients with type 2 diabetes and compared this with the hypothetically similar extent of impairments in patients with coronary artery disease (CAD).

Subjects and methods This case-control study (66 patients) compared four age-matched groups: control, type 2 diabetic, $\mathrm{CAD}$, and diabetic subjects with CAD (DCAD) and left ventricular ejection fraction $>50 \%$. CAD patients had $1-2$ vessel disease. Diastolic and systolic myocardial velocities were assessed with pulsed tissue Doppler. Global myocardial perfusion was assessed with contrast echocardiography as indices of capillary blood volume and myocardial blood flow at maximal vasodilatation. In CAD and DCAD patients, functional and perfusion parameters were additionally assessed in the territory with a normal coronary angiogram
\end{abstract}

\section{J. Jensen}

Department of Cardiology, Karolinska Hospital,

Stockholm, Sweden

V. Dounis

Department of Cardiology, General Air Force Hospital, Athens, Greece

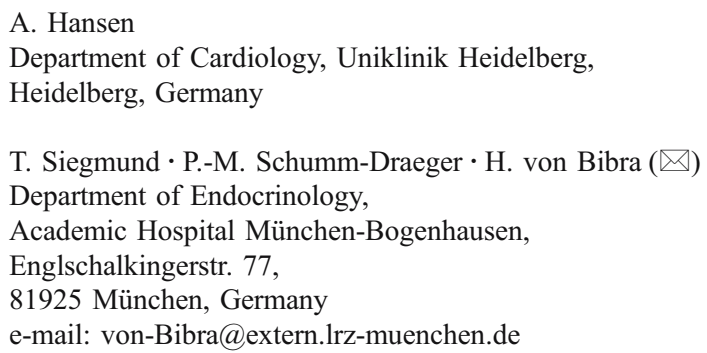

reading, providing a model for comparison with the global data from control and diabetic patients.

Results Comparing diabetic with control subjects, myocardial velocity at early diastole was impaired $(8.8 \pm 1.8$ vs $10.1 \pm 1.7 \mathrm{~cm} / \mathrm{s} ; p=0.02$ ) and correlated inversely with age, $\mathrm{HbA}_{1 \mathrm{c}}$ and pulse pressure $\left(R^{2}=0.761\right)$. Capillary blood volume (16.6 \pm 5.0 vs $24.4 \pm 4.9 \%$ ) and blood flow ( $56 \pm 35$ vs $114 \pm 40)$ were decreased $(p=0.001)$. In CAD patients, myocardial velocity at early diastole was similarly decreased $(p=0.02)$. CAD and DCAD patients were receiving more cardiovascular preventive therapy for the same extent of impaired global perfusion as in the less extensively treated diabetes group without CAD $(p<0.002)$, but had superior perfusion of the 'normal' coronary territory than that group $(p<0.05)$.

Conclusions/interpretation In patients with diabetes, global diastolic function and myocardial capillary blood volume and blood flow are impaired to the same extent as in patients with CAD. These impairments could form the basis of new therapeutic concepts.

Keywords Contrast echocardiography .

Coronary artery disease $\cdot$ Diastolic function .

Echocardiography $\cdot$ Myocardial perfusion .

Tissue Doppler · Type 2 diabetes mellitus
Abbreviations
CAD coronary artery disease
CBVI capillary blood volume index
DCAD type 2 diabetes mellitus combined with coronary artery disease
LV left ventricular
MBFI myocardial blood flow index
SI signal intensity 
Va late diastolic myocardial velocity

Ve early diastolic myocardial velocity

Vs systolic myocardial velocity

\section{Introduction}

A re-definition of the relationship between type 2 diabetes mellitus and coronary artery disease (CAD) [1] and heart failure is taking place, based on the combined incidence of cardiovascular disease and disturbed glucose metabolism $[2,3]$. Indeed, hyperglycaemia is increasingly being recognised as an equivalent to CAD [4]. This raises the question of whether type 2 diabetes in its present definition [5] and CAD stem from common pathophysiological mechanisms. If this is the case, one might expect both diseases to also be associated with similar myocardial disturbances in the essential performances of function and perfusion.

CAD is associated with impaired filling properties of the myocardium and with reduced myocardial perfusion $[6,7]$. Both abnormalities have also been observed in patients with type 1 and type 2 diabetes using techniques like traditional echocardiography [8,9], angiography and positron emission tomography [10 12]. However, there is a lack of evidence-based treatment strategies for these abnormalities in the diabetic heart, probably because the techniques mentioned above are not well suited for sequential studies after pharmacological intervention. However, the recent introduction of tissue Doppler allows serial quantification of systolic and diastolic myocardial function in patients with and without overt heart disease [7, 13, 14], and myocardial contrast echocardiography combined with contrast-specific imaging modalities $[15,16]$ allows repetitive quantification of myocardial perfusion $[17,18]$ and capillary blood volume [18-20].

Despite the growth of scientific knowledge of the close relation between CAD and disturbed glucose metabolism, the majority of general practitioners, as well as specialists in internal medicine and diabetes, still consider a patient with type 2 diabetes as a normal cardiac entity, unless there are clinically obvious signs and symptoms. The aim of this study, therefore, was to quantify myocardial function and perfusion abnormalities in the myocardium of patients with type 2 diabetes, who had near-normal glucose metabolism and no symptoms of heart failure, in comparison with patients with established CAD (1-2 vessel disease), with a view to testing the hypothesis that the extent of impairments is similar in both diseases.

\section{Subjects and methods}

Subjects

The extent of impairment of global myocardial diastolic function and of global microvascular perfusion was compared in four age-matched groups: control subjects $(n=14)$, type 2 diabetic $(n=17), 1-2$ vessel non-diabetic CAD $(n=17)$ and type 2 diabetic patients with $1-2$ vessel CAD (DCAD) $(n=18)$. Accordingly, a total of 66 patients was recruited from outpatients attending the Karolinska hospital: 35 patients with type 2 diabetes and 31 nondiabetic control subjects. Inclusion criteria were: (1) a left ventricular (LV) ejection fraction of $>50 \%$ (modified Simpson's method); and (2) sinus rhythm. Exclusion criteria were: microvascular complications, other than mild ones, of type 2 diabetes; CAD with 3 vessel disease; a history of previous myocardial infarction; history of LV failure; valvular heart disease other than mild; malignant hypertension; LV wall thickness $>13 \mathrm{~mm}$; obstructive airways disease; hepatic disease, other than mild (alanine aminotransferase $>59 \mathrm{U} / 1$ ); renal disease, other than mild (serum creatinine $>130 \mu \mathrm{mol} / \mathrm{l}$ ); or advanced malignancy. Coronary artery disease, defined as a $>50 \%$ reduction of the lumen in the proximal or mid-arterial tree, was assessed by coronary angiography in the 35 patients with CAD and DCAD. It was excluded by coronary angiography in 11 individuals and by traditional stress echocardiography [21] in the 20 remaining subjects who lacked clinical signs and symptoms and a history of coronary disease. We decided to define diabetes in this comparative study as use of antidiabetic medication or a fasting plasma glucose concentration $>7.0 \mathrm{mmol} / 1$ [5], because this is still a generally employed technique for the screening of diabetes.

Table 1 presents the demographic data, metabolic data, cardiovascular risk factors and cardiac medication in these four groups.

Ethics approval

The Ethics Committee of the Karolinska Hospital, Stockholm, approved the study protocol and all participants gave written informed consent to the study, which was designed in accordance with the Declaration of Helsinki.

\section{Study protocol}

Patients were studied after an overnight fast and without their morning medication by blood tests and ultrasound during the morning period. Resting blood pressure was measured to the nearest $2 \mathrm{mmHg}$ with a mercury sphygmomanometer with the patient in the supine position 
Table 1 Pertinent patient characteristics in all patients

\begin{tabular}{|c|c|c|c|c|c|c|c|}
\hline Characteristic & Control & Diabetes & $p$ value & $\mathrm{CAD}$ & $p$ value & DCAD & $p$ value \\
\hline$n$ & 14 & 17 & & 17 & & 18 & \\
\hline Age (years) & $58 \pm 7$ & $59 \pm 7$ & & $60 \pm 8$ & & $59 \pm 8$ & \\
\hline Male $(\%)$ & 71 & 71 & & 82 & & 88 & \\
\hline BMI $\left(\mathrm{kg} / \mathrm{m}^{2}\right)$ & $27 \pm 4$ & $28 \pm 4$ & & $28 \pm 4$ & & $29 \pm 4$ & \\
\hline Hyperlipidaemic (\%) & 14 & 43 & & $76^{\mathrm{a}}$ & 0.002 & 67 & 0.009 \\
\hline Hypertension (\%) & 21 & 41 & & 41 & & 58 & 0.024 \\
\hline Smoker (\%) & 36 & 47 & & 29 & & 25 & \\
\hline Beta blocker (\%) & 15 & 25 & & $94^{\mathrm{c}}$ & 0.001 & $72^{\mathrm{c}}$ & 0.001 \\
\hline ACE inhibitors (\%) & 8 & 25 & & 35 & & 28 & \\
\hline Aspirin $(\%)$ & 23 & 25 & & $94^{\mathrm{c}}$ & 0.001 & $67^{\mathrm{b}}$ & 0.005 \\
\hline Statins $(\%)$ & 0 & 38 & 0.02 & $76^{\mathrm{b}}$ & 0.001 & 50 & 0.002 \\
\hline Triglycerides $(\mathrm{mmol} / \mathrm{l})$ & $1.5 \pm 0.6$ & $2.7 \pm 2.6$ & & $1.8 \pm 1.0$ & & $1.9 \pm 1.3$ & \\
\hline Cholesterol (mmol/l) & $4.4 \pm 0.7$ & $5.2 \pm 1.2$ & 0.05 & $4.7 \pm 0.8$ & & $4.8 \pm 1.0$ & \\
\hline HDL (mmol/l) & $1.4 \pm 0.4$ & $1.0 \pm 0.3$ & 0.04 & $1.3 \pm 0.4$ & & $1.2 \pm 0.4$ & \\
\hline LDL (mmol/l) & $2.6 \pm 0.5$ & $3.1 \pm 1.0$ & & $2.5 \pm 0.7^{\mathrm{a}}$ & & $2.7 \pm 0.7$ & \\
\hline Fasting glucose $(\mathrm{mmol} / \mathrm{l})$ & $5.9 \pm 0.6$ & $9.8 \pm 2.8$ & n.a. & $6.1 \pm 0.5^{\text {n.a. }}$ & & $8.8 \pm 2.5$ & n.a. \\
\hline $\mathrm{HbA}_{1 \mathrm{c}}(\%)$ & $5.8 \pm 0.4$ & $6.7 \pm 1.1$ & 0.007 & $5.9 \pm 0.4^{\mathrm{b}}$ & & $6.4 \pm 1.2$ & 0.04 \\
\hline
\end{tabular}

With the exception of $n$, all values are means \pm SD. $p$ values are from post hoc test and denote significance vs control subjects.

$C A D$ Coronary artery disease, $D C A D$ diabetes with CAD, n.a. not applicable

a $p<0.05$

${ }^{\mathrm{b}} p<0.01$

${ }^{\mathrm{c}} p<0.001$ for significance vs diabetes

after a 5-min rest. Heart rate, blood pressure, ECG and ultrasound were recorded at rest and during hyperaemic stress (dipyridamole $0.84 \mathrm{mg} / \mathrm{kg}$ ). Two ultrasound systems were placed at either side of the patient and preset for tissue Doppler (System FiVe VingMed; General Electrics, Milwaukee, WI, USA) or myocardial contrast echocardiography (HDI 5000; ATL, Bothell, WA, USA). Myocardial velocity measurement was selected as a parameter of myocardial dysfunction because strain and strain rate were less efficient in assessing diastolic dysfunction in subclinical disease $[14,22]$. By nature, myocardial blood flow index (MBFI) and capillary blood volume index (CBVI), as measured by contrast echocardiography, are relative data and do not measure perfusion in $\mathrm{ml}$ per gram tissue. They do, however, allow comparative and non-ionising evaluation of myocardial perfusion [16-20].

This study on myocardial function and perfusion compares the effects of type 2 diabetes, as distinct from those of $\mathrm{CAD}$, which is a regional macrovascular disease. Adequate comparison, therefore, must focus on global LV perfusion and function parameters in analogy to the well-accepted assessment of LV ejection fraction. Additionally, the study design acknowledged the regional character of CAD. Thus, according to the inclusion criterion for 1-2 vessel disease, all CAD and DCAD patients had one angiographically normal coronary artery. Accordingly, perfusion data from the three to five segments composing this 'normal' territory were averaged to serve as a model for comparison with the situation in the control and type 2 diabetes groups without macrovascular disease.

Tissue Doppler

Pulsed Doppler myocardial velocity tracings were recorded as previously described [13, 23]. In short, the Doppler sampling gate was consecutively placed at the very basal and the mid position of each wall whilst imaging the apical four-, two- and three-chamber views. Thus, 12 myocardial velocity traces of longitudinal LV function were recorded in the septal, anteroseptal, anterior, lateral, posterior and inferior wall. According to individual coronary arterial distribution, these segments were combined to reflect the territories of the coronary arteries. With typical distribution of coronary arteries, the apical septum, anterior septal and anterior segments were ascribed to the left anterior descending artery, basal septum and inferior segments to the right coronary artery, and lateral and posterior segments to the circumflex artery [24]. The following peak velocities were determined from three consecutive cardiac cycles: systolic myocardial velocity (Vs), early diastolic myocardial velocity (Ve) and late diastolic myocardial velocity (Va). Patient-based global myocardial function was derived as an average value from the 12 regions. Additionally in CAD and DCAD patients, the myocardial velocities of the 'normal' coronary territory, as defined by angiography, were averaged from the respective three-five segments as $\mathrm{Vs}_{\text {norm }}, \mathrm{Ve}_{\text {norm }}$ and $\mathrm{Va}_{\text {norm }}$. 
Myocardial contrast echocardiography

Myocardial contrast echocardiography was performed as recently described $[16,20]$ using a broadband transducer at $1.77 \mathrm{MHz}$, pulse inversion grey scale imaging at a mechanical index of 1.2 and an intravenous infusion of the galactose-based contrast agent Levovist (Schering, Berlin, Germany) at $600 \mathrm{mg} / \mathrm{min}$ for $7 \mathrm{~min}$. Five endsystolic, triggered, two-dimensional images were recorded at incremental microbubble replenishment times of one, two, three, four, six and eight cardiac cycles in the apical four- and two-chamber view.

Using a dedicated image analysis program (HDI Lab version 1.83; ATL, Bothell, WA, USA), quantitative analysis of the digital images was performed by experienced observers (V. Dounis, H. von Bibra) who were unaware of the patients' clinical data. Background subtracted signal intensity (SI) was measured by densitometry (arbitrary units) in large myocardial regions of interest and in the LV cavity (Fig. 1). The relation between SI and the increasing trigger intervals $[17,20]$ allowed the calculation of the initial slope, which correlates to flow velocity, and of $\mathrm{SI}_{\text {plateau, }}$ which correlates to myocardial capillary blood volume. The

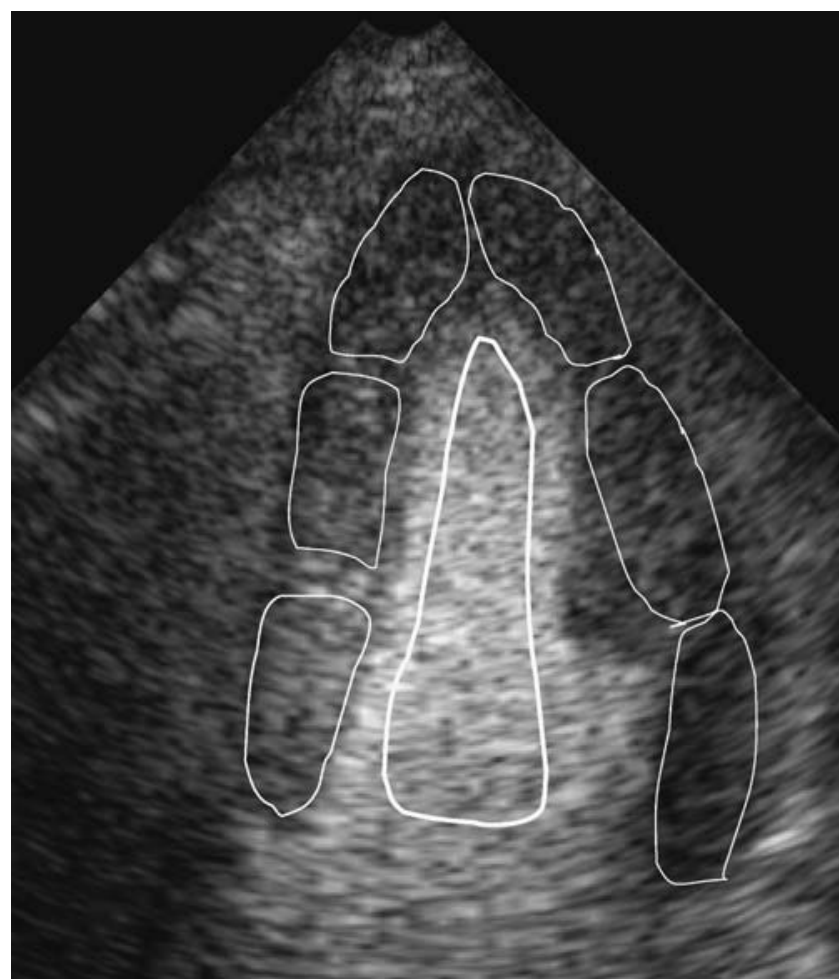

Fig. 1 An original example of myocardial perfusion imaging in the four-chamber view demonstrating six large regions of interest encompassing the myocardium for the assessment of global myocardial perfusion parameters as averaged value from all myocardial regions of interest. The signal intensity of these regions of interest is normalised for the instantaneous signal intensity in the left ventricular blood pool
CBVI was calculated as the mean value of SI at six and eight cardiac cycles replenishment time and normalised for the instantaneous signal intensity in the LV blood pool (\%). The MBFI was calculated as CBVI multiplied by the initial slope and coronary flow reserve index as the quotient of the respective values during stress and rest. The global perfusion parameters CBVI, flow velocity index and MBFI were averaged from the 12 regions of interest in each patient. Additionally, in the CAD and DCAD individuals, the perfusion parameters $\mathrm{CBVI}_{\text {norm }}$, initial slope ${ }_{\text {norm }}$ and $\mathrm{MBFI}_{\text {norm }}$ were averaged from the segments composing the 'normal' coronary territory.

\section{Statistical methods}

Sample size calculation: based on previous results [20,23], a clinically relevant effect size of 1.5 between two groups was assumed. A sample size of 14 in each group will have $80 \%$ power to detect an effect size of 1.5 using a two-group $t$-test with a 0.008 two-sided significance level. The level of significance was reduced according to Bonferroni's correction for multiple testing. Statistical analysis was performed using SPSS version 11.5. Data are expressed as mean \pm standard deviation in text, tables and plots. The four study groups (control, type 2 diabetes, CAD and DCAD) were compared with respect to parameters of myocardial perfusion and function using one-way ANOVA followed by post hoc least squared differences to correct for multiple testing problems. The significance level was set for two-tailed tests at $p=0.05$. Linear regression analysis was performed by least squares fitting. Univariate and multivariate analysis were used in the pooled data to examine correlations between Ve, Vs or CBVI and potential metabolic predictors of impaired myocardial function or perfusion.

\section{Results}

Diabetes characteristics

Demographic data were comparable between type 2 diabetic and DCAD patients (Table 1). The duration of diabetes was $7 \pm 8$ and $7 \pm 6$ years in the type 2 diabetic and the DCAD groups. Antidiabetic treatment was insulin in 53 and $42 \%$ of patients, respectively, with a mean dose of $38 \pm 19$ and $41 \pm 26 \mathrm{U}$ per day. A subanalysis was performed for type 2 diabetic patients with insulin therapy vs those without insulin therapy and repeated for the pooled type 2 diabetic and DCAD patients, both of which demonstrated similar myocardial function and perfusion parameters for these two subgroups. Metformin was being taken by 29 and $46 \%$, and sulfonylurea by 65 and $63 \%$ of the diabetic patients, respectively. Microvascular complications were 
mild retinopathy in $29 \%$ of patients, mild nephropathy in $12 \%$ and mild polyneuropathy in $15 \%$, with no significant difference between the two groups.

Comparison of type 2 diabetes with control

In type 2 diabetes, Ve was reduced $(p=0.02)$ at rest and during hyperaemic stress (Table 2, Fig. 2).

At rest, global perfusion parameters were comparable between both groups. At maximal vasodilatation, the global CBVI was significantly lower (Table 2, Fig. 3) in type 2 diabetic patients due to a diminished increase during hyperaemic stress $(p=0.001)$. This was associated with a reduction of the indices of myocardial blood flow velocity, myocardial blood flow and coronary flow reserve. With respect to haemodynamic data, pulse pressure was increased ( $p=0.05$, Fig. 2 ), as was the rate-pressure product at stress $(p=0.05$, Table 3$)$.
Comparison of CAD with control

In patients with CAD, Ve was reduced to a similar extent as in type 2 diabetic patients, both in global myocardial function and in the 'normal' territory (Fig. 2), at rest and at stress $(p=0.001)$. Vs was reduced $(p=0.008)$.

At maximal vasodilatation, all indices of global myocardial perfusion were reduced in comparison with control subjects, the reduction being to the same extent as in type 2 diabetic patients (Table 2). In the 'normal' territory, however, the indices of regional perfusion were comparable with control subjects (Fig. 3) and superior to global perfusion data in type 2 diabetes $(p=0.03)$. Of haemodynamic criteria, heart rate was lower $(p=0.04)$ than in control and type 2 diabetic patients. CAD patients had hyperlipidaemia more often $(p=0.009)$ and, as could be expected, were using more cardiovascular drugs, e.g. beta blockers $(p=0.001)$, aspirin $(p=0.001)$, and statins $(p=0.002$; Table 1$)$,

Table 2 Global and regional function and perfusion in the four groups of patients

\begin{tabular}{|c|c|c|c|c|c|c|c|c|}
\hline Parameter & & Control & Diabetes & $p$ value & CAD & $p$ value & DCAD & $p$ value \\
\hline$n$ & & & 14 & 17 & & 17 & & 18 \\
\hline \multirow[t]{2}{*}{ Vs resting $(\mathrm{cm} / \mathrm{s})$} & Global & $7.1 \pm 1.1$ & $6.5 \pm 1.4$ & & $6.0 \pm 1.1$ & 0.008 & $6.1 \pm 0.8$ & 0.02 \\
\hline & 'Normal' & & & & $6.5 \pm 1.2$ & & $6.6 \pm 1.0$ & \\
\hline \multirow[t]{2}{*}{ Vs s $(\mathrm{cm} / \mathrm{s})$} & Global & $7.7 \pm 1.4$ & $7.4 \pm 1.6$ & & $6.9 \pm 1.4$ & & $6.5 \pm 0.9$ & 0.03 \\
\hline & 'Normal' & & & & $7.4 \pm 1.2$ & & $7.3 \pm 1.2$ & \\
\hline \multirow[t]{2}{*}{ Ve resting $(\mathrm{cm} / \mathrm{s})$} & Global & $10.1 \pm 1.7$ & $8.8 \pm 1.8$ & 0.02 & $7.7 \pm 1.2^{\mathrm{a}}$ & 0.001 & $7.6 \pm 1.4^{\mathrm{b}}$ & 0.001 \\
\hline & 'Normal' & & & & $8.1 \pm 1.3$ & 0.001 & $8.1 \pm 1.9$ & 0.001 \\
\hline \multirow[t]{2}{*}{ Ve s $(\mathrm{cm} / \mathrm{s})$} & Global & $11.0 \pm 2.2$ & $9.5 \pm 1.7$ & 0.03 & $8.4 \pm 1.3^{\mathrm{a}}$ & 0.001 & $8.4 \pm 1.3^{\mathrm{a}}$ & 0.001 \\
\hline & 'Normal' & & & & $8.7 \pm 1.5$ & 0.001 & $9.4 \pm 1.7$ & 0.02 \\
\hline \multirow[t]{2}{*}{ Va resting $(\mathrm{cm} / \mathrm{s})$} & Global & $9.0 \pm 1.5$ & $10.0 \pm 2.0$ & & $7.9 \pm 1.6^{\mathrm{b}, \mathrm{d}}$ & & $9.1 \pm 1.8$ & \\
\hline & 'Normal' & & & & $8.5 \pm 1.8^{\mathrm{a}, \mathrm{d}}$ & & $10.1 \pm 2.2$ & \\
\hline \multirow[t]{2}{*}{ Va s $(\mathrm{cm} / \mathrm{s})$} & Global & $9.1 \pm 1.9$ & $10.2 \pm 2.0$ & & $8.7 \pm 1.6^{\mathrm{b}}$ & & $9.7 \pm 1.8$ & \\
\hline & 'Normal' & & & & $9.1 \pm 1.7^{\mathrm{a}, \mathrm{d}}$ & & $10.6 \pm 2.3$ & 0.05 \\
\hline e-a resting $(\mathrm{cm} / \mathrm{s})$ & & $1.1 \pm 2.1$ & $-1.2 \pm 2.7$ & 0.02 & $-0.2 \pm 2.0$ & & $-1.5 \pm 2.6$ & 0.004 \\
\hline $\mathrm{e}-\mathrm{a} \mathrm{s}(\mathrm{cm} / \mathrm{s})$ & & $1.8 \pm 3.3$ & $-1.3 \pm 2.8$ & 0.003 & $-0.3 \pm 1.6$ & 0.03 & $-1.3 \pm 2.2$ & 0.001 \\
\hline \multirow[t]{2}{*}{ CBVI s (\%) } & Global & $24.4 \pm 4.9$ & $16.6 \pm 5.0$ & 0.001 & $19.4 \pm 4.1^{\mathrm{d}}$ & 0.005 & $16.1 \pm 4.0$ & 0.001 \\
\hline & 'Normal' & & & & $25.9 \pm 6.0^{\mathrm{c}, \mathrm{d}}$ & & $21.4 \pm 4.2^{\mathrm{b}}$ & \\
\hline \multirow[t]{2}{*}{ Slope s } & Global & $4.6 \pm 1.0$ & $3.1 \pm 1.3$ & 0.001 & $3.3 \pm 0.8$ & 0.003 & $3.0 \pm 1.2$ & 0.001 \\
\hline & 'Normal' & & & & $4.0 \pm 0.7^{\mathrm{a}}$ & & $3.3 \pm 1.4$ & 0.003 \\
\hline \multirow[t]{2}{*}{ MBFI s } & Global & $114 \pm 40$ & $56 \pm 35$ & 0.001 & $65 \pm 25$ & 0.001 & $53 \pm 30$ & 0.001 \\
\hline & 'Normal' & & & & $100 \pm 31^{\mathrm{c}}$ & & $71 \pm 36$ & 0.002 \\
\hline \multirow[t]{2}{*}{$\Delta \mathrm{CBVI}$} & Global & $8.2 \pm 2.4$ & $2.2 \pm 2.9$ & 0.001 & $4.0 \pm 4.4$ & 0.001 & $2.7 \pm 3.3$ & 0.001 \\
\hline & 'Normal' & & & & $8.5 \pm 7.2^{\mathrm{b}}$ & & $7.8 \pm 5.4^{\mathrm{b}}$ & \\
\hline \multirow[t]{2}{*}{ CFRI } & Global & $2.9 \pm 0.9$ & $1.7 \pm 0.9$ & 0.04 & $2.6 \pm 2.1$ & & $2.0 \pm 1.4$ & \\
\hline & 'Normal' & & & & $3.6 \pm 3.1$ & & $4.5 \pm 4.0^{\mathrm{b}}$ & \\
\hline
\end{tabular}

With the exception of $n$, all values are means \pm SD. $p$ values are from post hoc test and denote significance vs control subjects.

$D C A D$ Diabetes and CAD, $s$ dipyridamole stress, $V s$ systolic velocity, Ve early diastolic velocity, Va late diastolic velocity, $e-a$ early minus late diastolic velocities, $C B V I$ capillary blood volume index, Slope index of myocardial blood flow velocity, $M B F I$ myocardial blood flow index, $\triangle C B V I$ stress-induced increase of capillary blood volume index, $C F R I$ coronary flow reserve index

${ }^{\mathrm{a}} p<0.05$

${ }^{\mathrm{b}} p<0.01$

${ }^{c} p<0.001$ for significance vs diabetes

d $p<0.05$ for significance vs DCAD 


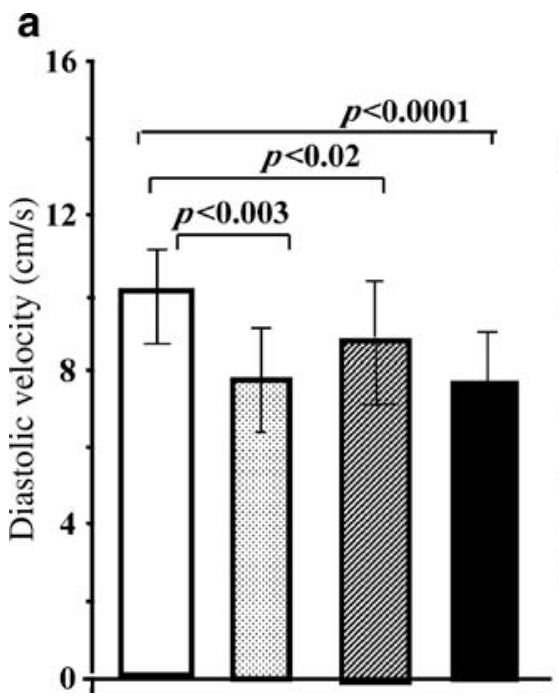

b

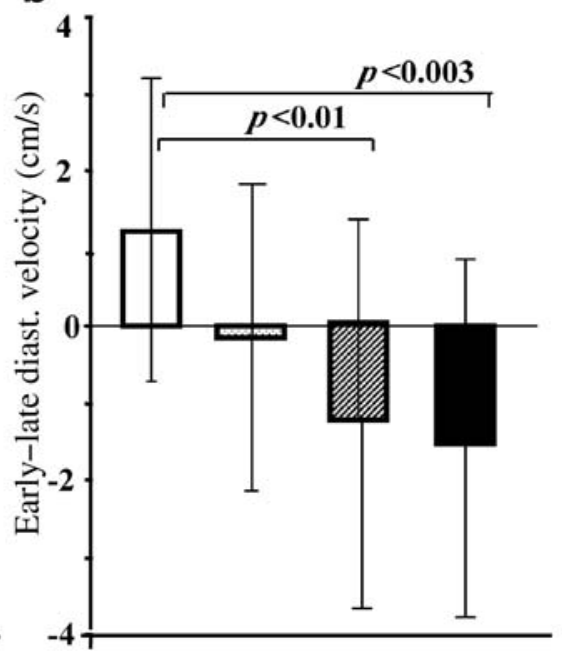

C

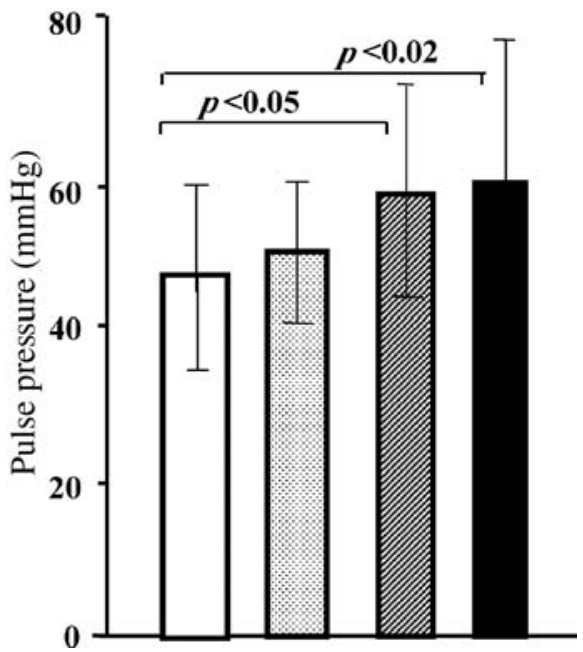

Fig. 2 Function-related parameters in the four groups: a early diastolic velocity; b early diastolic minus late diastolic velocity; and c pulse pressure. Control, white bars; CAD, dotted bars; diabetes without $\mathrm{CAD}$, hatched bars; diabetes with CAD, black bars. The reduction of early diastolic velocity was similar in the CAD and

than control and type 2 diabetic patients. However, lipid values and $\mathrm{HbA}_{1 \mathrm{c}}$ were comparable to control.

Comparison of DCAD with control

In patients with CAD and diabetes, Ve was reduced at rest and dipyridamole stress both in global function (Fig. 2) and diabetic groups. Only in the diabetes groups was it associated with an increase of late diastolic velocity so that the normal diastolic pattern, early diastolic $>$ late diastolic velocity, was inverted compared to the control. Pulse pressure was increased in both diabetic groups, but not in the CAD group

in the 'normal' territory (Table 2). Vs was reduced $(p=0.02)$.

All global myocardial perfusion indices at rest were comparable to the other three groups. At stress, they were reduced to a similar extent as in type 2 diabetic and CAD patients (Fig. 3). However, the indices of the 'normal' perfusion territory were comparable with control (Fig. 3) and significantly superior to type 2 diabetic
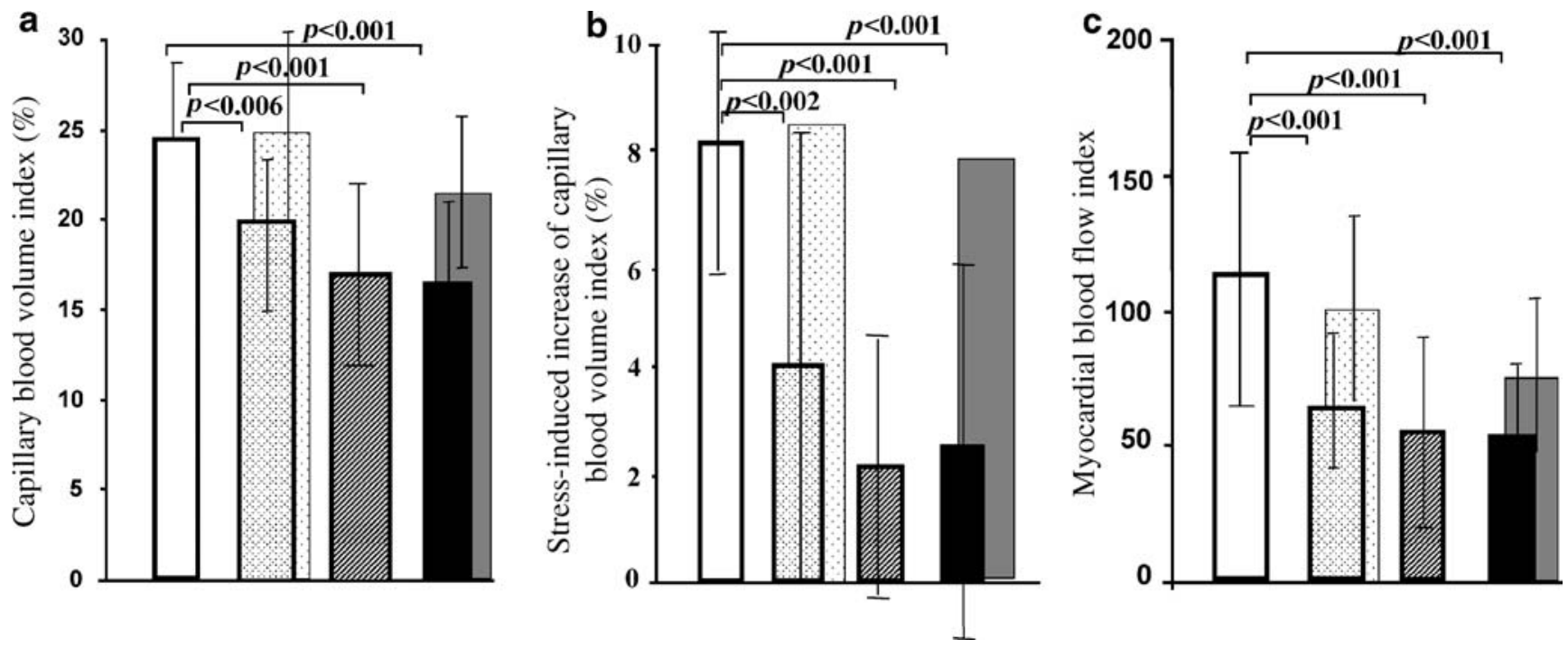

Fig. 3 The following perfusion indices during maximal vasodilatation in the four groups are shown: global capillary blood volume (a); global stress-induced increase of capillary blood volume index (b); and global myocardial blood flow index (c). Control, white bars; $\mathrm{CAD}$, dotted bars; diabetes without $\mathrm{CAD}$, hatched bars; diabetes with CAD, black bars. Partially concealed bars, values of the 'normal' territory for superimposed darker bars. Global CBVI and $\triangle \mathrm{CBVI}$ and MBFI were reduced in all patients' groups, see $p$ values. The flow indices $\mathrm{CBVI}_{\text {norm }}$ and $\mathrm{MBFI}_{\text {norm }}$ from the normal coronary territory in the $\mathrm{CAD}$ and, in particular, in the DCAD group were not different from control subjects. $\mathrm{CBVI}_{\text {norm }}$ was higher in DCAD $(p<0.006)$ and in CAD $(p<0.02)$ than CBVI in type 2 diabetes 
Table 3 Haemodynamic data of all patients

\begin{tabular}{|c|c|c|c|c|c|c|c|}
\hline Parameter & Control & Diabetes & $p$ value & CAD & $p$ value & DCAD & $p$ value \\
\hline$n$ & 14 & 17 & & 17 & & 18 & \\
\hline HR, resting (bpm) & $65 \pm 10$ & $68 \pm 10$ & & $57 \pm 10^{\mathrm{b}}$ & 0.04 & $64 \pm 10$ & \\
\hline $\mathrm{HR}, \mathrm{s}(\mathrm{bpm})$ & $81 \pm 12$ & $82 \pm 9$ & & $77 \pm 11$ & & $78 \pm 12$ & \\
\hline BP systolic, resting $(\mathrm{mmHg})$ & $125 \pm 14$ & $136 \pm 14$ & & $127 \pm 14$ & & $137 \pm 23$ & 0.06 \\
\hline BP systolic $\mathrm{s}(\mathrm{mmHg})$ & $115 \pm 11$ & $134 \pm 13$ & 0.001 & $127 \pm 15$ & 0.04 & $135 \pm 19$ & 0.001 \\
\hline BP diastolic resting $(\mathrm{mmHg})$ & $77 \pm 6$ & $81 \pm 9$ & & $77 \pm 8$ & & $73 \pm 11$ & \\
\hline $\mathrm{BP}$ diastolic s (mmHg) & $69 \pm 11$ & $77 \pm 9$ & & $73 \pm 7^{\mathrm{b}}$ & & $68 \pm 10^{\mathrm{a}}$ & \\
\hline $\mathrm{RPP}$ resting $(\mathrm{mmHg} / \mathrm{min})$ & $8,192 \pm 1,888$ & $9,583 \pm 1,887$ & 0.05 & $7,318 \pm 1,563^{\mathrm{b}, \mathrm{c}}$ & & $8,780 \pm 1,868$ & \\
\hline RPP s (mmHg/min) & $9,310 \pm 1,797$ & $10,850 \pm 1,704$ & 0.05 & $10,036 \pm 2,250$ & & $10,514 \pm 2,111$ & \\
\hline PP resting $(\mathrm{mmHg})$ & $48 \pm 14$ & $58 \pm 15$ & 0.05 & $51 \pm 10^{\mathrm{c}}$ & & $63 \pm 18$ & 0.007 \\
\hline PP s (mmHg) & $46 \pm 8$ & $60 \pm 11$ & 0.003 & $53 \pm 12^{d}$ & & $67 \pm 16$ & 0.001 \\
\hline
\end{tabular}

With the exception of $n$, all values are means \pm SD. $p$ values are from post hoc test and denote significant differences vs control subjects.

$C A D$ Coronary artery disease, $D C A D$ diabetes and CAD, $s$ dipyridamole stress, $H R$ heart rate, $B P$ blood pressure, $R P P$ rate pressure product,

$P P$ pulse pressure

${ }^{\mathrm{a}} p<0.05$

${ }^{\mathrm{b}} p<0.01$ for significance vs diabetes

${ }^{\mathrm{c}} p<0.05$

d $p<0.01$ for significance vs DCAD

patients $(p=0.006)$. Expectedly, cardiovascular drugs were used more intensively, e.g. beta blockers $(p=0.001)$, aspirin $(p=0.005)$ and statins $(p=0.002$, Table 1$)$, in comparison with the control and type 2 diabetes groups ( $p=0.001,0.005$ and 0.012 , respectively). Within regard to haemodynamics, pulse pressure was increased ( $p=0.007$, Table 3$)$. Additionally, hypertension and hyperlipidaemia were more frequent $(p=0.03$ and $p=0.009$, respectively).

Control and type 2 diabetes subgroups

without hypertension

In order to avoid the impact of hypertensive heart disease in control and type 2 diabetic patients, subgroups were established for patients in these groups with resting blood pressure $\leq 140 / 90 \mathrm{mmHg}$ and no antihypertensive treatment $(n=10$ in control and in type 2 diabetes groups). Similar decreases of $\mathrm{Ve}(p=0.05)$, impairment of all parameters of perfusion $(p=0.02)$ and increase of pulse pressure $(p=0.05)$ were observed in the diabetic subgroup, this being associated with a similar distribution of clinical data and cardiac medication as demonstrated in Table 1 for type 2 diabetes and control (Table 4).

Correlations for parameters of function and perfusion

The pooled data of the four groups demonstrated significant inverse correlations of myocardial function or perfusion values with metabolic parameters as detailed in Table 5 . Diabetes duration, triglycerides and total cholesterol did not correlate with parameters of function or perfusion. When all the associated factors for Ve were entered into a forward stepwise regression model, the results showed only $\mathrm{HbA}_{1 \mathrm{c}}$, $\mathrm{Vs}$ and pulse pressure to be independent predictors $\left(R^{2}=0.761\right)$.

There were similar correlations for the combined control and type 2 diabetes groups as detailed in Table 5, but even closer inverse correlations between $\mathrm{Ve}$ and age or $\mathrm{HbA}_{1 \mathrm{c}}$, HDL and the daily dose of insulin. Similarly, CBVI had closer inverse correlations with BMI or with the daily dose of insulin, systolic blood pressure and $\mathrm{HbA}_{1 \mathrm{c}}$.

\section{Discussion}

Using modern ultrasound technology, abnormalities of global myocardial perfusion and diastolic function were quantified and compared between patients with type 2 diabetes and age-matched individuals without and with 1-2 vessel CAD. We found that Ve was reduced in the type 2 diabetes group by the same extent as in the CAD group, and that the reduction correlated with pulse pressure, which was increased. Global perfusion parameters were markedly reduced at maximal vasodilatation to the same extent as in the CAD and DCAD groups. Both of the latter were using more beta blockers, aspirin and statins than type 2 diabetes or control patients, and this was associated with improved perfusion data of the 'normal' coronary territory in DCAD patients compared to the type 2 diabetic group.

Myocardial dysfunction in type 2 diabetes

The well-known diastolic dysfunction of patients with diabetes mellitus $[8,9,20,25]$ was confirmed by our data 
Table 4 Control and type 2 diabetes subgroups of individuals without hypertension
With the exception of $n$, all values are means $\pm \mathrm{SD}$.

$B P$ blood pressure, $C B V I$ capillary blood volume index, $H R$

Heart rate, $M B F I$ myocardial blood flow index, $P P$ pulse pressure, $R P P$ rate pressure product, $s$ dipyridamole stress, Slope index of myocardial blood flow velocity

\begin{tabular}{lllll}
\hline Characteristic & & Control & Diabetes & $p$ value \\
\hline$n$ & & & 10 & \\
Age & $($ years $)$ & $59 \pm 8$ & $60 \pm 7$ & \\
HbA $_{1 \mathrm{c}}$ & $(\%)$ & $5.6 \pm 0.4$ & $7.0 \pm 1.2$ & \\
BMI & $\left(\mathrm{kg} / \mathrm{m}^{2}\right)$ & $26 \pm 3$ & $47 \pm 5$ & 0.06 \\
Statins & $(\%)$ & 0 & $2.2 \pm 1.0$ & \\
Triglycerides & $(\mathrm{mmol} / \mathrm{l})$ & $1.4 \pm 0.6$ & $5.1 \pm 0.9$ & \\
Cholesterol & $(\mathrm{mmol} / \mathrm{l})$ & $4.2 \pm 0.7$ & $1.1 \pm 0.4$ & \\
HDL & $(\mathrm{mmol} / \mathrm{l})$ & $1.4 \pm 0.4$ & $3.4 \pm 0.9$ & \\
LDL & $(\mathrm{mmol} / \mathrm{l})$ & $2.6 \pm 0.6$ & $68 \pm 12$ & \\
HR & $(\mathrm{bpm})$ & $64 \pm 10$ & $82 \pm 11$ & 0.05 \\
BP systolic & $(\mathrm{mmHg})$ & $120 \pm 10$ & $9,674 \pm 2,082$ & 0.05 \\
BP diastolic & $(\mathrm{mmHg})$ & $77 \pm 6$ & $61 \pm 17$ & \\
RPP & $(\mathrm{mmHg} / \mathrm{min})$ & $7,711 \pm 1,606$ & $1.0 \pm 0.3$ & 0.03 \\
PP & $(\mathrm{mmHg})$ & $43 \pm 11$ & $3.1 \pm 1.0$ & 0.005 \\
Vs & $(\mathrm{mmol} / \mathrm{l})$ & $1.4 \pm 0.4$ & $3.0 \pm 1.5$ \\
Ve & $(\mathrm{mmol} / \mathrm{l})$ & $2.6 \pm 0.5$ & $17.9 \pm 5.0$ & 0.007 \\
Slope s & & $4.6 \pm 1.2$ & $58 \pm 41$ & 0.003 \\
CBVI s & $(\%)$ & $23.7 \pm 5.3$ & & \\
MBFI s & & $110 \pm 45$ & & \\
\hline
\end{tabular}

from tissue Doppler-derived velocity measurements of LV long axis function during early diastole, a finding that is consistent with other reports $[14,20,26]$. Diastolic dysfunction has been mainly attributed to structural changes of the diabetic myocardium [27, 28] but there is also increasing evidence that metabolic [29] and other dynamic changes are involved, e.g. mismatch of oxygen demand and supply [13] and modification of afterload, tissue perfusion, possibly endothelial function [8,30], and finally antidiabetic therapy $[20,30]$. As early diastolic function is energyconsuming, it can be speculated that limited availability of metabolic substrates for energy production on the basis of impaired tissue perfusion contributes to the underlying pathophysiology. Abnormal early diastolic function relates to impaired vital prognosis $[31,32]$, but evidence-based medicine still lacks studies for effective therapy.

Despite the basic and close correlation between systolic and diastolic function, systolic longitudinal LV function was not significantly impaired in our patients. In the early stage of diabetic heart disease, this may be related to compensation by increased short-axis LV function [14, 26], and also to an increase of late diastolic filling velocities, which counterbalances the reduction in early diastolic relaxation properties of the diabetic myocardium, thereby maintaining global filling and, thus, systolic function. Our observation in the present type 2 diabetes group was in line with this latter mechanism (Fig. 2). The observed mechanisms of diastolic dysfunction in patients with CAD were different and were associated with impaired systolic function.
Diastolic dysfunction correlated to pulse pressure

The magnitude of early Ve in type 2 diabetic and control groups correlated inversely with age, confirming earlier reports $[23,33]$. In this context, it can be speculated that stiffening of the arterial wall contributes to diastolic dysfunction, as it is the leading vascular abnormality of old age and diabetes mellitus [34]. Indeed, pulse pressure, a surrogate of arterial stiffening, was significantly elevated only in the groups with diabetic individuals (Fig. 2), and was confirmed as an independent predictor for disturbed Ve. Increasing pulse pressure is known to impair the functional interaction between central arterial wall and left ventricle [35], partially due to reduced myocardial perfusion pressure and elevated endsystolic afterload [36, 37]. The benefits of therapeutic afterload reduction are known in diabetic patients [38]. Arterial stiffening, therefore, could conceivably become a target of therapeutic strategies in the treatment of diastolic dysfunction. In patients with type 2 diabetes, this is in line with optimising glucose metabolism as suggested by the inverse correlation between $\mathrm{Ve}$ and $\mathrm{HbA}_{1 \mathrm{c}}$.

\section{Diastolic dysfunction in CAD patients}

Expectedly, global early diastolic function was reduced in CAD patients (Fig. 2). This observation has been attributed to ischaemia-induced regional asynchrony of myocardial contraction and relaxation [6,7]. Although the magnitude 
Table 5 Pearson correlation coefficients of significant correlations

\begin{tabular}{|c|c|c|c|c|c|c|}
\hline \multirow[t]{2}{*}{ Parameter } & \multirow[t]{2}{*}{ Pooled data from } & \multicolumn{2}{|c|}{ Myocardial function } & \multicolumn{3}{|c|}{ Indices of myocardial perfusion } \\
\hline & & Vs & $\mathrm{Ve}$ & Flow velocity & Capillary blood volume & Blood flow \\
\hline \multirow{2}{*}{ Age } & 4 groups & n.s. & -0.441 & n.s. & n.s & n.s \\
\hline & $\mathrm{C}+\mathrm{D}$ & n.s. & -0.515 & n.s. & n.s. & n.s. \\
\hline \multirow[t]{2}{*}{ Systolic blood pressure } & 4 groups & n.s & -0.239 & n.s & n.s & n.s \\
\hline & $\mathrm{C}+\mathrm{D}$ & n.s. & n.s. & -0.381 & -0.433 & -0.459 \\
\hline \multirow[t]{2}{*}{ Pulse pressure } & 4 groups & n.s. & -0.260 & n.s. & n.s. & n.s. \\
\hline & $\mathrm{C}+\mathrm{D}$ & n.s & n.s & n.s & n.s & n.s \\
\hline \multirow[t]{2}{*}{ Rate pressure product } & 4 groups & n.s. & n.s. & n.s. & n.s. & n.s \\
\hline & $\mathrm{C}+\mathrm{D}$ & n.s & n.s & n.s & n.s & -0.359 \\
\hline \multirow[t]{2}{*}{ BMI } & 4 groups & n.s. & n.s. & n.s. & -0.295 & -0.268 \\
\hline & $\mathrm{C}+\mathrm{D}$ & n.s. & n.s. & n.s. & -0.485 & -0.374 \\
\hline \multirow[t]{2}{*}{$\mathrm{HbA}_{1 \mathrm{c}}$} & 4 groups & n.s. & -0.365 & n.s. & n.s. & n.s. \\
\hline & $\mathrm{C}+\mathrm{D}$ & n.s. & -0.449 & n.s. & -0.401 & -0.427 \\
\hline \multirow[t]{2}{*}{ Insulin (units/day) } & 4 groups & n.s. & n.s. & n.s. & -0.393 & n.s. \\
\hline & $\mathrm{C}+\mathrm{D}$ & n.s. & -0.538 & n.s. & -0.486 & -0.428 \\
\hline \multirow[t]{2}{*}{ HDL } & 4 groups & n.s. & n.s. & n.s. & n.s. & n.s. \\
\hline & $\mathrm{C}+\mathrm{D}$ & n.s. & -0.422 & n.s. & n.s. & n.s. \\
\hline \multirow[t]{2}{*}{ LDL } & 4 groups & n.s. & n.s. & -0.286 & n.s. & n.s. \\
\hline & $\mathrm{C}+\mathrm{D}$ & n.s. & n.s. & -0.438 & n.s. & n.s. \\
\hline \multirow[t]{2}{*}{ Vs } & 4 groups & & 0.632 & n.s. & n.s. & n.s. \\
\hline & $\mathrm{C}+\mathrm{D}$ & & 0.672 & n.s. & n.s. & n.s. \\
\hline \multirow[t]{2}{*}{$\mathrm{Ve}$} & 4 groups & 0.632 & & n.s. & n.s. & n.s. \\
\hline & $\mathrm{C}+\mathrm{D}$ & 0.672 & & n.s. & n.s. & n.s. \\
\hline \multirow{2}{*}{ Slope hyperaemic stress } & 4 groups & n.s. & n.s. & & 0.749 & 0.932 \\
\hline & $\mathrm{C}+\mathrm{D}$ & n.s. & n.s. & & 0.796 & 0.946 \\
\hline \multirow{2}{*}{ CBVI, hyperaemic stress } & 4 groups & n.s. & n.s. & 0.749 & & 0.909 \\
\hline & $\mathrm{C}+\mathrm{D}$ & n.s. & n.s. & 0.796 & & 0.922 \\
\hline \multirow[t]{2}{*}{ MBFI, hyperaemic stress } & 4 groups & n.s. & n.s. & 0.932 & 0.909 & \\
\hline & $\mathrm{C}+\mathrm{D}$ & n.s. & n.s. & 0.946 & 0.922 & \\
\hline
\end{tabular}

$C$ Control, $C B V I$ capillary blood volume index, $D$ type 2 diabetes, $M B F I$ myocardial blood flow index $=$ flow velocity index $\times$ capillary blood volume index, n.s. no correlation at significant level $(p<0.05)$, Slope index of myocardial blood flow velocity

of the decrease in Ve was similar to that observed in type 2 diabetes patients, it was not associated with a compensatory increase of $\mathrm{Va}$, but with significantly reduced systolic function, reflecting different pathophysiological mechanisms than in the type 2 diabetes group.

Myocardial perfusion in type 2 diabetic patients

The adaptive modifications of tissue perfusion to metabolic demands are based on dynamic capillary blood volume changes transmitted by recruitment or derecruitment of capillaries [16-20, 39, 40]. It has not been shown before that patients with diabetes had markedly reduced capillary blood volume during maximal vasodilatation (Fig. 3) irrespective of the presence or absence of hypertension. Notably, it was the stress-induced percentage increase of capillary volume that was predominantly reduced (17 vs $50 \%$ in control subjects). These data suggest that the diabetic myocardium at hyperaemic stress cannot recruit sufficient additional capillaries to facilitate metabolic exchange. This limitation is particularly disadvantageous, as the diabetic heart works on a higher level of oxygen demand than the heart of CAD patients, at least as reflected by the rate-pressure product. Our observation that myocardial blood flow during stress and coronary flow reserve are also reduced is in line with the above findings, confirming observations by PET and angiography [10-12].

A noteworthy observation, and one not shown before, was that global perfusion in patients with macrovascular CAD was impaired to the same extent as in diabetic patients with microvascular disease (Fig. 3). These microvascular abnormalities are probably linked to endothelial dysfunction, which is known to deteriorate with hyperglycaemia and other cardiovascular risk factors [40-42] as confirmed in the present data by the inverse correlations between myocardial perfusion and metabolic and haemodynamic parameters (Table 5). However, the interaction between endothelial dysfunction and microvascular perfusion abnormalities also contains dynamic and reversible components. Indeed, first reports have been published on 
improvement of myocardial perfusion by short-term pharmacological action [20, 30, 43].

In this context, the present data demonstrate some therapeutic potential in diabetic patients. Thus the global perfusion parameters and surrogate parameters for myocardial oxygen demand were similar between the type 2 diabetes and DCAD groups. However, the perfusion values of the 'normal' vascular territory were significantly superior in DCAD than in type 2 diabetic patients and no longer different from control subjects and CAD patients (Fig. 3). This was associated with significantly increased use of beta blockers, aspirin and statins in the DCAD group, so that interpretation of the origin of flow enhancement is limited. Further prospective studies are needed to evaluate the effects of preventive therapy on the reversible components of impaired perfusion in the diabetic heart. The potential of antidiabetic therapy was also confirmed in the present data by the inverse correlation between MBFI and $\mathrm{HbA}_{1 \mathrm{c}}$, which was significant ( $p=0.007$, Table 5$)$ in the combined type 2 diabetes and control groups.

Prediabetes and metabolic syndrome

The striking likeness of global perfusion impairment between patients with macrovascular CAD versus patients with type 2 diabetes and microvascular abnormalities was based on the definition of diabetes according to the established diagnostic marker of fasting serum glucose $\geq 7 \mathrm{mmol} / \mathrm{l}$ [5]. There is a large body of evidence with convincingly similar results worldwide, which suggests a fairly high prevalence (around 51-63\%) of impaired glucose regulation in patients with $\mathrm{CAD}$ and non-diabetic fasting glucose values [3, 44-46]. Moreover, the increased but similar BMI between our CAD and type 2 diabetes patients suggests a high and similar prevalence of metabolic syndrome or insulin resistance in both age-matched groups. It can be assumed, therefore, that the similar impairment of myocardial perfusion in both groups is related, in the majority of patients, to the disturbed glucose metabolism and/or insulin resistance that are typical of the metabolic syndrome, all of which have been shown to be associated with endothelial dysfunction [41, 47]. This is in line with the significant and inverse correlations of perfusion data with BMI in the pooled data of all groups. The present data thus support the 'common soil' theory for diabetes mellitus and coronary artery disease and point to important options for preventive therapy.

\section{Study limitations}

The numbers of patients in the four groups were small but sufficient to yield statistically meaningful results. According to recent surveys $[44,48,49]$, the definition of diabetes on the basis of an additional OGTT could have led to 0.7 persons (5\%) in the control group and 2.4 patients (14\%) in the CAD group being diagnosed with diabetes mellitus. This, however, is unlikely to weaken our very significant differences between control subjects and the three patient groups, since exclusion of false negative patients in CAD would instead increase the power of the test.

All diabetes-specific observations were confirmed in each of the diabetic groups, irrespective of CAD or hypertensive effects. The effects from abnormal serum viscosity and red blood cell rheology or microangiopathy cannot be differentiated from the more dynamic impairment of capillary recruitment during stress observed by us. The normal range of perfusion parameters in the resting state, however, points to the predominant influence of the latter mechanism.

In the CAD groups, cardiac medication created an additional influence to the effects of the disease. Obviously, the present study of function and perfusion could not be designed without the effects of chronic cardiac or antidiabetic medication, as it would have been unethical to stop these. The evaluation of the specific effects of the respective medications used by these patients should be undertaken in future prospective studies. Most diabetic patients without CAD were not on cardiac medication. In the DCAD group, however, it cannot be ruled out that some beneficial effects of cardiac therapy masked the expected increasingly detrimental effects of combined diabetes and CAD (Figs. 2 and 3).

Finally, a more uniform application of angiography for the exclusion of coronary disease would have been warranted, but was not clinically applicable for ethical reasons in asymptomatic diabetic and non-diabetic patients. However, the exclusion of CAD by traditional 2-dimensional stress echocardiography was supported by a simultaneous lack of stress-induced myocardial velocity reduction in the combined traditional echocardiographic and tissue Doppler stress test [13].

\section{Clinical implications}

The present study in patients with type 2 diabetes demonstrates new findings with potentially important therapeutic implications:

(1) Impaired diastolic myocardial velocity is inversely related to age, $\mathrm{HbA}_{1 \mathrm{c}}$ and pulse pressure.

(2) Myocardial capillary volume at maximal vasodilatation is reduced leading to an impairment of global myocardial blood flow.

(3) The observed limitations of microvascular perfusion in patients with diabetes mellitus are similar in extent to the macro/microvascular abnormalities in coronary 
artery disease, but may apparently be compensated by the use of preventive cardiovascular therapy, an optimisation of glucose metabolism or other ways of ameliorating endothelial function, all of which appear promising as therapeutic targets. Similarly, diastolic dysfunction appears to be related to arterial stiffening, also a potentially important therapeutic target.

Acknowledgements This study was supported by grants from the Swedish Heart and Lung Foundation and funds from the Karolinska Institute for H. von Bibra. V. Dounis was supported by an educational grant from the Hellenic air forces. A. Hansen received an educational grant from the German Cardiac Society.

\section{References}

1. DECODE study group on behalf of the European Diabetes Epidemiology Group (1999) Glucose tolerance and mortality: comparison of WHO and American Diabetic Association diagnostic criteria. Lancet 354:617-621

2. Kannel WB, McGee DL (1979) Diabetes and cardiovascular disease. The Framingham Study. J Am Med Assoc 214:20352038

3. Norhammar A, Tenerz A, Nilsson G, Hamsten A, Efendic S, Rydén L (2003) Glucose metabolism in patients with acute myocardial infarction and no previous diagnosis of diabetes mellitus: a prospective study. Lancet 359:2140-2144

4. Grundy SM, Benjamin IJ, Burke GL et al (1999) Diabetes and cardiovascular disease. AHA scientific statement for healthcare professionals from the American Heart Association. Circulation 100:1134-1146

5. Expert Committee on the Diagnosis and Classification of Diabetes Mellitus (1997) Report of the Expert Committee on the diagnosis and classification of diabetes mellitus. ADA Diabetes classification (2005). Diabetes Care 28(Suppl 1):1-79

6. Doran JH, Trail TA, Brown DJ, Gibson DG (1978) Detection of abnormal left ventricular wall movement during isovolumic contraction and early relaxation. Br Heart J 40:367-371

7. Garcia MJ, Thomas JD, Klein AL (1998) New Doppler echocardiographic applications for the study of diastolic function. J Am Coll Cardiol 32:865-875

8. Celentano A, Vaccaro O, Tammaro P et al (1995) Early abnormalities of cardiac function in non-insulin-dependent diabetes mellitus and impaired glucose tolerance. Am J Cardiol 76:1173-1176

9. Liu JE, Palmieri V, Roman MJ et al (2001) The impact of diabetes on left ventricular filling pattern in normotensive and hypertensive adults: the Strong Heart Study. J Am Coll Cardiol 37:1943-1949

10. Nahser PJ, Brown RE, Oskarsson H et al (1995) Maximal coronary flow reserve and metabolic coronary vasodilatation in patients with diabetes. Circulation 91:6635-6640

11. Strauer BE, Motz W, Vogt M, Schwartzkopf B (1997) Impaired coronary flow reserve in NIDD. A possible role for diabetic cardiomyopathy in humans. Diabetes 46(Suppl 2):119-124

12. Pitkänen O-P, Nuutila P, Raitakari OT et al (1998) Coronary flow reserve is reduced in young men with IDD. Diabetes 47:248-254

13. von Bibra H, Tuchnitz A, Klein A et al (2000) Regional diastolic function by pulsed Doppler myocardial mapping for the detection of left ventricular ischaemia during pharmacologic stress testing- a comparison with stress echocardiography and perfusion scintigraphy. J Am Coll Cardiol 36:444-452

14. Fang ZY, Yuda S, Anderson V et al (2003) Echocardiographic detection of early diabetic myocardial disease. J Am Coll Cardiol 41:611-617

15. Porter TR, Xie F, Silver M et al (2001) Real-time perfusion imaging with low mechanical index pulse inversion Doppler imaging. J Am Coll Cardiol 37:748-753

16. von Bibra H, Bone D, Niklasson U et al (2002) Myocardial contrast echocardiography yields best accuracy using quantitative analysis of digital data from pulse inversion technique comparison with second harmonic imaging and harmonic power Doppler during simultaneous dipyridamole stress SPECT studies. Eur J Echocardiog 3:271-282

17. Wei K, Jayaweera AR, Firoozan S et al (1998) Quantification of myocardial blood flow with ultrasound-induced destruction of microbubbles administered as a constant venous infusion. Circulation 97:473-483

18. Dawson D, Vincent MA, Barrett EJ et al (2002) Vascular recruitment in skeletal muscle during exercise and hyperinsulinaemia assessed by contrast ultrasound. Am J Physiol Endocrinol Metab 282:E714-E720

19. Wu CC, FelDan MD, Mills JD et al (1997) Myocardial contrast echocardiography can be used to quantify intramyocardial blood volume: new insights into structural mechanisms of coronary autoregulation. Circulation 96:1004-1011

20. Hansen A, Johansson BL, Wahren J, von Bibra H (2002) Beneficial effects of C-peptide on myocardial function in patients with type 1 diabetes. Diabetes 51:3077-3082

21. American Society of Echocardiography Committee on Standards, subcommittee on Quantification of Two Dimensional Echocardiograms (1989) Recommendations for the left ventricle by two dimensional echocardiography. J Am Soc Echocardiog 2:258-267

22. Koyama J, Ray-Sequin PA, Falk RH (2003) Longitudinal myocardial function assessed by tissue velocity, strain and strain rate tissue Doppler echocardiography in patients with $\mathrm{AL}$ (primary) cardiac amyloidosis. Circulation 107:2446-2452

23. von Bibra H, Thrainsdottir IS, Hansen A, Dounis V, Malmberg K, Rydén L (2005) Tissue Doppler imaging for the detection and quantitation of myocardial dysfunction in patients with type 2 diabetes mellitus: a methodological study. Diabetes Vasc Dis Res 2:483-487

24. Lang RM, Bierig M, Devereux RB et al (2006) Recommendations for chamber quantification. Eur J Echocardiog 7:79-108

25. Jain A, Avendano G, Dharamsey S et al (1996) Left ventricular diastolic function in hypertension and role of plasma glucose and insulin. Comparison with the diabetic heart. Circulation 93:13961402

26. Vinereanu D, Nicolaides E, Tweddel A et al (2003) Subclinical left ventricular dysfunction in asymptomatic patients with type 2 diabetes mellitus, related to serum lipids and glycated haemoglobin. Clin Sci 2003 105:591-599

27. Brownlee M (1992) Glycation products and the pathogenesis of diabetic complications. Diabetes Care 15:1835-1843

28. Mizushige K, Yao L, Noma $T$ et al (2000) Alteration in left ventricular diastolic filling and accumulation of myocardial collagen at insulin-resistant prediabetic stage of a type 2 diabetic rat model. Circulation 101:899-907

29. Diamant M, Lamb HJ, Groeneveld Y et al (2003) Diastolic dysfunction is associated with altered myocardial metabolism in asymptomatic normotensive patients with well-controlled type 2 diabetes mellitus. J Am Coll Cardiol 42:328-335

30. von Bibra H, Hansen A, Dounis V, Bystedt T, Malmberg K, Rydén L (2004) Insulin based improved metabolic control augments myocardial diastolic function and perfusion in patients with type 2 diabetes mellitus. Heart 90:1483-1484 
31. Bella JN, Palmieri V, Roman MJ et al (2002) Mitral ratio of peak early to late diastolic filling velocity as a predictor of mortality in middle-aged and elderly adults. The Strong Heart Study. Circulation 105:1928-1933

32. Wang M, Yip G, Wanf A et al (2003) Peak early diastolic mitral annulus velocity by tissue Doppler imaging adds independent and incremental prognostic value. J Am Coll Cardiol 43:820-826

33. Henein M, Lindqvist P, Francis D et al (2002) Tissue Doppler analysis of age-dependency in diastolic ventricular behaviour and filling. A cross-sectional study of healthy hearts (the Umea General Population Heart Study). Eur Heart J 23:162-171

34. Lakatta EG, Levy D (2003) Arterial and cardiac aging: major shareholders in cardiovascular disease enterprises. Part 1: Aging arteries: a set up for vascular disease. Circulation 107:139-148

35. Dart AM, Kingwell BA (2001) Pulse pressure: a review of mechanisms and clinical relevance. J Am Coll Cardiol 37: 975-984

36. Leite-Moreira AF, Correia-Pinto J, Gillebert TC (1999) Afterload induced changes in myocardial relaxation. A mechanism for diastolic dysfunction. Cardiovasc Res 43:344-533

37. Vinereanu D, Nicolaides E, Boden L et al (2003) Conduit arterial stiffness is associated with impaired left ventricular subendocardial function. Heart 89:449-450

38. Heart outcomes prevention evaluation (HOPE) study investigators (2000) Effects of ramipril on cardiovascular and microvascular outcomes in people with diabetes mellitus: results of the HOPE study and MICRO-HOPE substudy. Lancet 355:253-259

39. Vincent MA, Dawson D, Clark A et al (2002) Skeletal muscle microvascular recruitment by physiological hyperinsulinaemia precedes increases in total blood flow. Diabetes 51:42-48

40. Wiernsperger NF (2001) In defence of microvascular constriction in diabetes. Clin Hemorheol Microcirc 25:55-62
41. Williams SB, Goldfine AB, Timimi FK et al (1998) Acute hyperglycaemia attenuates endothelium-dependent vasodilatation in humans in vivo. Circulation 97:1695-1701

42. Ceriello A, Taboga C, Tonuti L et al (2002) Evidence for an independent and cumulative effect of postprandial hypertriglyceridaemia and hyperglycaemia on endothelial dysfunction and oxidative stress generation. Circulation 106:1211-1218

43. Rask-Madsen C, Ihleman N, Krarup T et al (2001) Insulin therapy improves insulin-stimulated endothelial function in patients with type 2 diabetes and ischemic heart disease. Diabetes 50:2611-2618

44. Bartnik M, Malmberg K, Norhammar A, Tenerz A, Öhrvik J, Rydén L (2004) Newly detected abnormal glucose tolerance: an important predictor of long-term outcome after myocardial infarction. Eur Heart J 25:1990-1997

45. Satoh H, Terada H, Uehara A et al (2005) Post-challenge hyperinsulinaemia rather than hyperglycaemia is associated with the severity of coronary artery disease in patients without a previous diagnosis of diabetes mellitus. Heart 91:731-736

46. Kowalska I, Prokop J, Bachorzewska-Gajewska H et al (2001) Disturbances of glucose metabolism in men referred for coronary arteriography. Diabetes Care 24:897-901

47. Wykretowicz A, Guzik P, Bartkowiak G et al (2005) Endothelial function and baroreflex sensitivity according to the oral glucose tolerance test in patients with coronary artery disease and normal fasting glucose levels. Clin Sci 109:397-403

48. Rathmann W, Haastert B, Icks A et al (2003) High prevalence of undiagnosed diabetes mellitus in southern Germany: target populations for efficient screening. the KORA survey. Diabetologia 46:182-189

49. Harris MI, Flegal K, Cowie CC et al (1998) Prevalence of diabetes, impaired fasting glucose and impaired glucose tolerance. Diabetes Care 21:518-524 